\title{
Modèles murins du SIDA : drapeau rouge
}

Plusieurs modèles murins de l'infection par HIV ont été décrits. Citons les souris transgéniques exprimant la totalité du génome viral et les souris immunodéprimées SCID (severe combined immunodeficiency) chez lesquelles peuvent être greffées des cellules sanguines humaines infectées par HIV ( $\mathrm{m} / \mathrm{s} n^{\circ} 10$, vol. 4, p. 658). L'intérêt de tels modèles est évidemment de pouvoir étudier, sur un animal de laboratoire facile à utiliser, toutes les phases de la maladie, et tester l'efficacité de nouvelles molécules ou combinaisons de molécules sur l'infection virale et ses conséquences. C'est ainsi que l'efficacité de l'AZT a été récemment démontrée chez des souris SCID greffées avec des cellules humaines infectées par HIV-1. Par ailleurs, on sait que la souris possède de nombreux rétrovirus endogènes, les uns ne constituant que des vestiges séquellaires d'infections passées et persistant sous la forme de séquences génomiques silencieuses et parfaitement stables, les autres susceptibles de s'exprimer. Or, c'est là que le bât blesse. En effet, les virus co-exprimés dans une même cellule sont susceptibles d'échanger du matériel (c'est même le principe de la complémentation en trans utilisée pour la préparation des vecteurs rétroviraux, ou de l'effet des virus auxiliaires sur la réplication des virus "défectifs"), voire de recombiner leurs génomes. Par exemple, deux virus peuvent échanger des portions de leurs protéines d'enveloppe dont dépend principalement le tropisme cellullaire. Afin de tester si de tels phénomènes pouvaient réellement être observés, l'équipe de R. Gallo (NCI, Bethesda, $\mathrm{MD}$, USA), associée à des chercheurs italiens de Turin et à une autre équipe américaine, a infecté par HIV-1 des cellules tumorales hématopoḯtiques humaines préalablement infectées par un virus endogène de souris, X-MuLV (xenotropic murine leukemia virus). Cette infection avait été obtenue in vivo par greffe de tissu tumoral humain à des souris athymiques Nude. Ces cellules $\mathrm{T}$ humaines exprimaient, avant et après passage dans la souris Nude, la protéine membranaire CD4 et pouvaient donc être aisément infectables par HIV-1. Cependant les cellules ayant acquis le virus xénotrope murin étaient le siège d'une infection beaucoup plus rapide et sévère que les cellules d'origine. D'autres tests confirmaient le rôle aggravant de X-MuLV sur l'infection de cellules humaines par HIV-1. Plus inquiétant encore, les particules virales produites par les cellules préalablement infectées par X-MuLV avaient un tropisme cellulaire très élargi : elles pouvaient infecter des cellules B et des cellules T CD4$\mathrm{CD}^{+}{ }^{+}$[1]. En d'autres termes, ces cellules produisaient un virus HIV-1 modifié plus infectieux sur un plus grand nombre de cellules.

On imagine sans peine ce que cela pourrait donner si ces résultats étaient transposables à la souris entière [2]. Ces travaux ont au moins deux types de conséquences. Le premier est que les mesures de précaution doivent être exceptionnelles puisque les souris "sidéennes " sont non seulement une source possible de dissémination du virus, mais aussi d'un virus de virulence peut-être accrue. Le second est qu'il faut rester prudent sur l'applicabilité à l'homme des résultats obtenus sur de tels modèles animaux, puisqu'il semble que leurs virus infectants aient pu acquérir des propriétés nouvelles. Gallo et ses collaborateurs suggèrent que l'infection systématique, par des rétrovirus endogènes, des cellules humaines greffées chez la souris SCID pourrait expliquer certaines particularités du système, telle l'absence de réaction du greffon contre l'hôte : les cellules $\mathrm{T}$ cytotoxiques $\mathrm{CD}^{+}{ }^{+}$seraient rendues anergiques par une telle infection.

C'est donc à une extraordinaire incitation à la prudence qu'aboutissent ces travaux: prudence quant à la possibilité de jouer les apprentis sorciers et d'engendrer des agents infectieux hypervirulents, réalisant en laboratoire ce qui a probablement été à l'origine, de façon spontanée dans la nature, de l'apparition des HIV actuels. Prudence aussi quant à la valeur des modèles animaux pour prédire l'évolution et la sensibilité aux médicaments de maladies humaines.

A.K.

1. Lusso P, Veronese FDM, Ensoli B, et al. Expanded HIV-1 cellular tropism by phenotypic mixing with murine endogenous retrovirus. Science 1990 ; 247 : 848-52.

2. Marx J. Concerns raised about mouse models for AIDS. Science $1990 ; 247: 809$. 